\title{
气相爆轰合成碳包铁的影响因素
}

\author{
间鸿浩 ${ }^{1}$, 赵铁军 ${ }^{1}$, 孙贵砳 ${ }^{3}$, 李晓杰 ${ }^{1,2}$, 浑长宏 ${ }^{1}$
}

(1. 大连理工大学 工程力学系, 大连 116024; 2. 大连理工大学 工业装备结构分析国家重点实验室, 大连 116024 ; 3. 中国劳动关系学院 安全工程系, 北京 100048)

摘 要: 研究了氢氧摩尔比例与二茂铁质量对制备碳包覆铁纳米颗粒的影响。通过氢气与氧气爆轰产生的高温高速, 在自制爆轰管内分解二茂铁，合成了碳包覆铁纳米颗粒。经 XRD 分析与 TEM 表征发现，氢氧比例与二茂铁质量在 制备碳包铁时相互影响，氢氧比例主要影响碳包铁的形貌大小及分散性，二茂铁质量对能否合成碳包铁纳米颗粒 影响很大。当氢氧比例为 $2: 1$, 二茂铁质量在 2.5 3.5 g 时, 能够合成碳包覆铁纳米颗粒, 且呈球形或椭球形, 具有 明显的核壳结构。从颗粒大小均匀性，形貌结构及分散性考虑，氢氧比例 $2: 1$ ，二茂铁质量 $3.5 \mathrm{~g}$ 的反应条件是制备 碳包铁的较佳方案。

关 键 词: 气相爆轰法; 碳包铁; 氢氧比例; 二茂铁

中图分类号: O389 文献标识码: A

\section{Influence Factors of Preparing Carbon-encapsulated Iron Nanoparticles by Gaseous Detonation Method}

\author{
YAN Hong-Hao ${ }^{1}$, ZHAO Tie-Jun ${ }^{1}$, SUN Gui-Lei ${ }^{3}$, LI Xiao-Jie ${ }^{1,2}$, HUN Chang-Hong ${ }^{1}$
}

(1. Department of Engineering Mechanics, Dalian University of Technology, Dalian 116024, China; 2. State Key Laboratory of Structural Analysis for Industrial Equipment, Dalian 116024, China; 3. Department of Safety Engineering, China Institute of Industrial Relations, Beijing, 100048, China)

\begin{abstract}
Effects of molar ratio of hydrogen to oxygen and ferrocene mass on synthesis of carbon-encapsulated iron nanoparticles (Fe@C) were studied. Fe@C were prepared by using ferrocene as material resource, which decomposed by high temperature and high speed caused by hydrogen and oxygen detonation in homemade detonation tube. The samples were characterized by X-ray diffraction, transmission electron microscope and high resolution transmission electron microscope and it can be found that the molar ratio of hydrogen to oxygen, and ferrocene mass interact each other in the preparation of Fe@C. The molar ratio of hydrogen to oxygen mainly influences the morphology, particle size and dispersion of Fe@C, while ferrocene mass has a great affect on the synthesis of Fe@C. Part of iron element will be oxidized at small dosage of ferrocene, on the contrary, ferrocene couldn't be decomposed completely. When the molar ratio of hydrogen to oxygen is $2: 1$, ferrocene mass ranges in 2.5-3.5 g, spherical or ellipsoidal like Fe@C is prepared with a clear core-shell structure, and thickness of carbon shell at about $10 \mathrm{~nm}$. In consideration of particle size uniformity, morphology structure and dispersion, the best condition for preparing Fe@C is, the molar ratio of hydrogen to oxygen at 2:1, ferrocene mass at $3.5 \mathrm{~g}$.
\end{abstract}

收稿日期: 2015-11-09; 收到修改稿日期：2015-12-12

基金项目: 国家自然科学基金(10872044,10602013, 10972051, 10902023); 辽宁省自然科学基金(20082161), 北京高等学校 青年英才计划

National Natural Science Foundation of China (10872044, 10602013, 10972051, 10902023); Natural Science Foundation of Liaoning Province, China (20082161); Beijing Higher Education Young Elite Teacher Project

作者简介：间鸿浩(1974-), 男, 博士，副研究员. E-mail: honghaoyan@vip.sina.com

通讯作者: 李晓杰, 教授. E-mail: 923309973@qq.com 
Key words: gaseous detonation method; carbon-encapsulated iron; hydrogen and oxygen ratio; ferrocene

碳包覆金属纳米颗粒具有特殊的核壳结构, 使 其具有特殊的电磁性 ${ }^{[1-2]}$ 、吸附性 ${ }^{[1]}$ 、保护纳米金属 ${ }^{[3]}$ 等特性, 在电子领域、污水处理、生物医学 ${ }^{[4]}$ 等领域 有很好的应用潜力。目前, 碳包覆金属纳米颗粒的

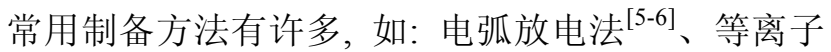
体法 ${ }^{[7]}$ 和 CVD 法 ${ }^{[8]}$ 等。但这些方法一般成本较高、 产率较低。Wu 等 ${ }^{[9]}$ 用苦味酸与二茂铁在钢制容器内 热解爆炸合成了碳包铁后, 爆轰法作为一种简单 快捷的碳包金属纳米颗粒的方法受到广泛重视。本 课题组先后研究了用爆轰法制备碳包铁 ${ }^{[10]}$ 、碳包 镍 ${ }^{[11-13]}$ 、碳包钴 ${ }^{[12-13]}$ 、碳包铜 ${ }^{[14]}$ 等纳米颗粒，并对 其形成机理做了一系列探究。上述研究都是采用凝 聚态炸药作为能量来源, 但凝聚态炸药对设备要 求较严格。为了解决该问题, 在爆轰法合成碳包金 属机理的基础上, 本工作用可燃性气体与氧气作 为爆源, 研究气相爆燃 (爆车) 制备碳包覆铁纳米颗 粒, 分析氢氧比例以及二茂铁质量对合成碳包铁 的影响。

\section{1 实验方法}

\section{1 实验原理}

氢气与氧气混合气体经火花塞起爆后, 首先发 生爆燃反应, 然后转变为爆轰反应。火焰传播速度 由亚音速向超音速转变, 且压力波以声速传播。温 度超过 $100^{\circ} \mathrm{C}$ 后, 二茂铁升华为气态, 可与氢氧气 体均匀混合。在满足爆燃向爆轰转变条件的爆轰管 内, 用火花塞引爆混合气体, 瞬时高温高速使二茂 铁分解，该过程的化学反应方程式为:

$$
\begin{gathered}
\left(\mathrm{C}_{5} \mathrm{H}_{5}\right)_{2} \mathrm{Fe}(\mathrm{s}) \rightarrow 10 \mathrm{C}(\mathrm{s})+5 \mathrm{H}_{2}(\mathrm{~g})+\mathrm{Fe}(\mathrm{s}) \\
2 \mathrm{H}_{2}(\mathrm{~g})+\mathrm{O}_{2}(\mathrm{~g}) \rightarrow 2 \mathrm{H}_{2} \mathrm{O}(\mathrm{g})
\end{gathered}
$$

式中, $\mathrm{s}$ 表示固体, $\mathrm{g}$ 表示气体。且在该过程中, 整个 化学反应应在负氧平衡下进行。

\section{2 气相爆轰合成碳包铁实验}

图 1 为实验装置, 爆轰管长 $1100 \mathrm{~mm}$, 内径 $95 \mathrm{~mm}$, 外径 $190 \mathrm{~mm}$, 体积为 $7.8 \mathrm{~L}$, 满足爆然向爆 轰转变的条件。将通入的气体看作理想气体, 根据 分压原理控制通入氢气与氧气的体积。实验中, 用

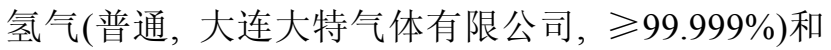
氧气(高纯，大连大特气体有限公司， $\geqslant 99.999 \%$ )作 为爆源, 二茂铁(分析纯, 天津致远化学试剂有限公 司, $\geqslant 99 \%)$ 作为碳源与铁源。

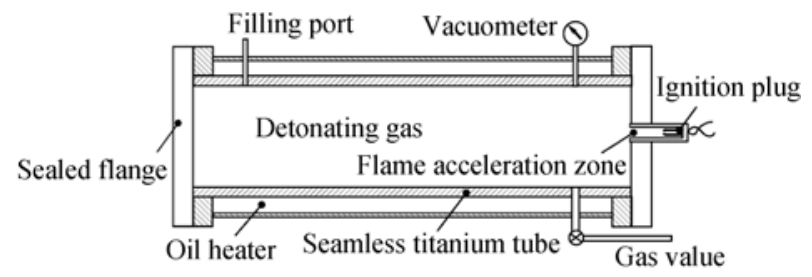

图 1 气相爆轰管的示意图

Fig. 1 Schematic of gaseous detonation tube

\subsection{1 改变氢氧比例}

取 $3.5 \mathrm{~g}$ 二茂铁放置在爆轰管末端，接着抽真空， 按表 1 通入一定量的氢气与氧气, 然后启动加热系 统，将温度保持在 $140^{\circ} \mathrm{C}$, 待气压表保持稳定后，用 起爆器点火。静置 $20 \mathrm{~min}$ 左右, 收集沉淀在管壁的 粉末。

\subsection{2 改变二茂铁质量}

该实验过程与不同氢氧比例实验相同，所需二 茂铁质量与爆轰反应初始条件如表 2 所示。

用 $\mathrm{X}$ 射线衍射仪(D/MAX-2400 型, $\mathrm{Cu} \mathrm{K} \alpha, \lambda=$ $0.15406 \mathrm{~nm}$ )和透射电子显微镜(Tecnai G220 S-twin 型, 美国 FEI 公司生产)对样品的晶型与形貌进行 表征。

\section{2 结果与讨论}

\section{1 氢氧比例对气相爆轰合成碳包铁的影响} 由凝聚态炸药爆轰合成碳包金属纳米颗粒可知,

表 1 不同氢氧比例的实验初始条件

Table 1 Conditions of initial experimental with different ratio of hydrogen to oxygen

\begin{tabular}{ccccc}
\hline Sample & $\begin{array}{c}\text { Tempera- } \\
\text { ture/ }{ }^{\circ} \mathrm{C}\end{array}$ & $\begin{array}{c}\text { Initial pres- } \\
\text { sure/MPa }\end{array}$ & $\begin{array}{c}\text { Ferrocene } \\
\text { mass } / g\end{array}$ & $n\left(\mathrm{H}_{2}\right): n\left(\mathrm{O}_{2}\right)$ \\
\hline $1 \#$ & 140 & 0.1 & 3.5 & $1: 1$ \\
$2 \#$ & 140 & 0.1 & 3.5 & $2: 1$ \\
\hline
\end{tabular}

表 2 不同二茂铁质量的实验初始条件

Table 2 Conditions of initial experimental with different ferrocene mass

\begin{tabular}{ccccc}
\hline Sample & $\begin{array}{c}\text { Tempera- } \\
\text { ture } /{ }^{\circ} \mathrm{C}\end{array}$ & $\begin{array}{c}\text { Initial pres- } \\
\text { sure/MPa }\end{array}$ & $\begin{array}{c}\text { Ferrocene } \\
\text { mass/g }\end{array}$ & $n\left(\mathrm{H}_{2}\right): n\left(\mathrm{O}_{2}\right)$ \\
\hline $1 \#$ & 120 & 0.1 & 1.5 & $2: 1$ \\
$2 \#$ & 120 & 0.1 & 2.5 & $2: 1$ \\
$3 \#$ & 120 & 0.1 & 3.0 & $2: 1$ \\
$4 \#$ & 120 & 0.1 & 3.5 & $2: 1$ \\
$5 \#$ & 120 & 0.1 & 4.0 & $2: 1$ \\
\hline
\end{tabular}


前驱体各元素间只有满足负氧平衡时，才有可能制 备出碳包覆金属纳米颗粒。从表 3 可看出, 氢氧摩 尔比分别为 $1: 1$ 与 $2: 1$ 时, 均满足负氧平衡, 符合爆 轰法制备碳包金属的基本条件。

图 2 是不同氢氧比例下所得样品的 XRD 图谱, 从图中可以看出, 在 $2 \theta=26.40^{\circ}$ 处有一个碳的衍射 峰, 从衍射峰的形状可以确定存在石墨型碳或者无 定型碳。样品 $1 \#$ 与 $2 \#$ 中, $2 \theta=44.48^{\circ} 、 43.27^{\circ}$ 处衍射 峰分别对应 $\mathrm{Fe}(110)$ 和 $\mathrm{Fe}_{3} \mathrm{C}(111)$ 晶面, 且 2 \#样品中 铁的衍射峰相对较强。 $\mathrm{Fe}_{3} \mathrm{C}$ 可能是爆车高温条件下 铁原子与碳原子发生化合反应生成的。

用 Scherrer 公式 $D=\frac{\mathrm{K} \lambda}{B \cos \theta}$ (式中, $\mathrm{K}$ 为常数, 取 $0.89 ; \lambda$ 为入射波长, 取 $0.15406 \mathrm{~nm}$; $B$ 为衍射半 峰宽; $\theta$ 为布拉格角)计算样品的平均晶粒度 $D$, 计 算结果见表 4 。从表 4 中可以看出, 1 \#样品的平均粒 径较 2\#样品大。

表 3 反应物中各元素的摩尔构成及其氧平衡

Table 3 Moles of each element in the reactants and the corresponding oxygen balance

\begin{tabular}{|c|c|c|c|c|c|c|}
\hline \multirow{2}{*}{ Sample } & \multicolumn{4}{|c|}{ Mole of each element } & \multirow{2}{*}{$n(\mathrm{H}): n(\mathrm{O})$} & \multirow{2}{*}{$\begin{array}{l}\text { Oxygen } \\
\text { balance }\end{array}$} \\
\hline & $\mathrm{H}$ & $\mathrm{O}$ & $\mathrm{C}$ & $\mathrm{Fe}$ & & \\
\hline $1 \#$ & 0.536 & 0.348 & 0.188 & 0.0188 & $1.5: 1.0$ & $<0$ \\
\hline $2 \#$ & 0.652 & 0.232 & 0.188 & 0.0188 & $2.8: 1.0$ & $<0$ \\
\hline
\end{tabular}

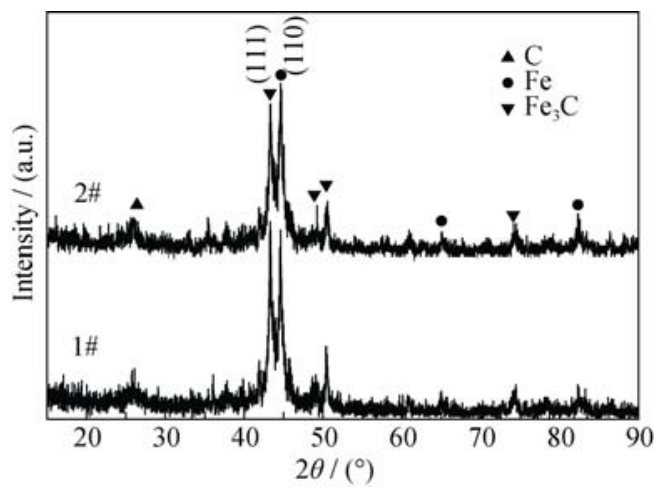

图 2 不同氢氧比例制备样品的 XRD 图谱

Fig. 2 XRD patterns of samples prepared with different ratio of hydrogen to oxygen

表 4 爆轰产物的平均粒径

Table 4 Average particle size of the detonation products

\begin{tabular}{ccccc}
\hline Sample & Phases & $2 \theta /\left(^{\circ}\right)$ & FWHM & $\begin{array}{c}\text { Mean grain } \\
\text { size, } D / \mathrm{nm}\end{array}$ \\
\hline \multirow{2}{*}{$1 \#$} & $\mathrm{Fe}$ & 44.5 & 0.274 & 31 \\
& $\mathrm{Fe}_{3} \mathrm{C}$ & 43.2 & 0.401 & 21 \\
\multirow{2}{*}{$2 \#$} & $\mathrm{Fe}$ & 44.5 & 0.374 & 23 \\
& $\mathrm{Fe}_{3} \mathrm{C}$ & 43.3 & 0.553 & 16 \\
\hline
\end{tabular}

图 3 是不同氢氧比例制备样品的 TEM 照片, 从图中可看出, $1 \#$ 样品与 2 \#样品均有球形或半球 形纳米颗粒生成，且有明显的核壳结构，结合 XRD 分析，可确定其为碳包覆铁或铁碳化合物纳 米颗粒 ${ }^{[5,9]}$ 。对比 A-1 与 B-1 可知, 1 \#样品分散性 较差, 2\#样品分散性较好。从 A-2 与 B-2 中可看出, $1 \#$ 样品的颗粒大小较为均匀，但碳壳结构并不完 善，尤其是游离碳加剧了样品的团聚; 2 \#样品的 核壳结构比较完整，颗粒大小在 10 30 nm 之间。 虽然颗粒大小不是很均匀，但具有良好的包覆效 果与较好的分散性。因此, 在该实验装置中, 较理 想的氢氧摩尔比为 $2: 1$ 。

\section{2 二茂铁质量对气相爆轰合成碳包铁的影响}

从表 5 能够看出，二茂铁质量分别为 $1.5 、 2.5$ 、 $3.0 、 3.5$ 和 $4.0 \mathrm{~g}$ 的实验中，也均满足负氧平衡，满 足制备碳包覆金属纳米颗粒的基本条件。
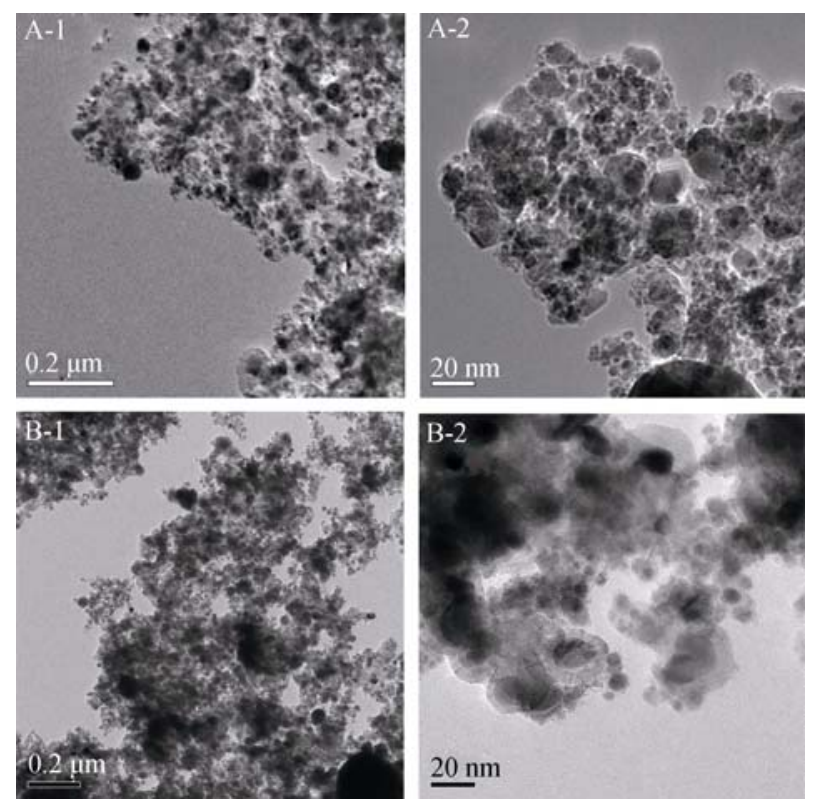

图 3 不同氢氧比例制备样品的 TEM 照片

Fig. 3 TEM images of samples prepared with different ratio of hydrogen to oxygen

Ratios of hydrogen to oxygen of A to B are 1:1, 2:1, respectively

表 5 反应物中各元素的摩尔构成及其氧平衡

Table 5 Moles of each element in the reactants and the corresponding oxygen balance

\begin{tabular}{ccccccc}
\hline \multirow{2}{*}{ Sample } & \multicolumn{5}{c}{ Mole of each element } & $n(\mathrm{H}): n(\mathrm{O}) \begin{array}{c}\text { Oxygen } \\
\text { balance }\end{array}$ \\
\cline { 2 - 7 } & $\mathrm{H}$ & $\mathrm{O}$ & $\mathrm{C}$ & $\mathrm{Fe}$ & & $<0$ \\
\hline $1 \#$ & 0.545 & 0.232 & 0.081 & 0.0081 & $2.3: 1$ & $<0$ \\
$2 \#$ & 0.598 & 0.232 & 0.134 & 0.0134 & $2.6: 1$ & $<0$ \\
$3 \#$ & 0.625 & 0.232 & 0.161 & 0.0161 & $2.7: 1$ & $<0$ \\
$4 \#$ & 0.652 & 0.232 & 0.188 & 0.0188 & $2.8: 1$ & $<0$ \\
$5 \#$ & 0.679 & 0.232 & 0.215 & 0.0215 & $2.9: 1$ & $<0$ \\
\hline
\end{tabular}


从 XRD 图谱(图 4)可以看出, 五个样品中均有 $\mathrm{Fe}$, 表明二茂铁均被分解。1\#样品中主要含有 $\mathrm{Fe}$ 与 $\mathrm{FeO}$, 未见碳以及铁碳化合物, 这主要是由于二茂 铁的含量太低, 在爆轰过程中氧气把二茂铁中的碳 全部氧化, 并且还有一部分剩余的氧气与二茂铁中 的铁发生反应, 并且为缺氧反应, 只能生成 $\mathrm{FeO} 。 2 \#$ 样品中 $\mathrm{FeO}$ 的衍射峰有所减弱, 出现 $\mathrm{Fe}_{3} \mathrm{C}$ 的衍射峰, 说明随着二茂铁质量的增加, 二茂铁分解产生多余 的碳, 在高温条件下可与铁发生反应。从 $3 \# 、 4 \#$ 以 及 5 \#样品的图谱中发现, 随着二茂铁质量的增加, XRD 图谱中铁碳化合物和氧化亚铁的衍射峰逐渐 减弱, 甚至消失, 而铁的衍射峰仍比较强。这是因为 随着二茂铁用量的增加, 氧气的相对含量逐渐减小, 从而与铁发生反应的氧气减少。其中 $4 \#$ 样品在 $2 \theta=26.40^{\circ}$ 左右出现的衍射峰源于石墨碳或者无定 形碳。在 $2 \theta=44.64^{\circ} 、 65.08^{\circ} 、 82.34^{\circ}$ 处衍射峰对应 体心立方结构纳米铁的(110)、(200)、(211)晶面。 $2 \theta=36.10^{\circ} 、 41.80^{\circ} 、 60.80^{\circ}$ 附近衍射峰对应 $\mathrm{FeO}$ 的 (111)、(200)、(220)晶面。

从表 6 可以看出, 随着二茂铁质量的增加, 平 均晶粒度呈逐渐下降趋势, 但规律性不明显, 可能 是由于环境的变化以及实验的条件变化造成的。

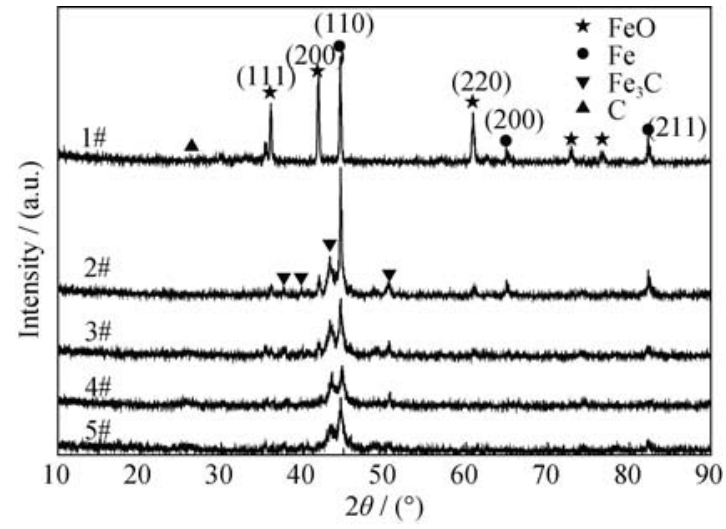

图 4 不同二茂铁质量合成产物的 XRD 图谱

Fig. 4 XRD patterns of detonation products with different ferrocene mass

Ferrocene mass of 1\#, 2\#, 3\#, 4\# and 5\# are 1.5, 2.5, 3.0, 3.5 and $4.0 \mathrm{~g}$, respectively

表 6 爆轰产物中铁颗粒的平均粒径

Table 6 Average particle size of iron in detonation products

\begin{tabular}{ccccc}
\hline Sample & Phases & $2 \theta /\left(^{\circ}\right)$ & FWHM & $\begin{array}{c}\text { Mean grain size, } \\
D / \mathrm{nm}\end{array}$ \\
\hline $1 \#$ & $\mathrm{Fe}$ & 44.5 & 0.169 & 50 \\
$2 \#$ & $\mathrm{Fe}$ & 44.6 & 0.275 & 30 \\
$3 \#$ & $\mathrm{Fe}$ & 44.6 & 0.221 & 38 \\
$4 \#$ & $\mathrm{Fe}$ & 44.5 & 0.374 & 23 \\
$5 \#$ & $\mathrm{Fe}$ & 44.6 & 0.264 & 32 \\
\hline
\end{tabular}

结合 1\#样品的 XRD 图谱(图 4)和 TEM 照片 (图 5)中的 A-1、A-2 可见，存在没有碳包覆纳米 颗粒，颗粒很大，图中为 $\mathrm{Fe}$ 颗粒或者 $\mathrm{FeO}$ 颗粒， 可见当二茂铁含量较少时，二茂铁分解产生的碳 几乎全部被氧化。从图 5 中 B-1、B-2 可发现, 产 物中均含有包覆层纳米结构, 结合前面的 XRD 图 谱分析可以确定包覆层纳米颗粒为碳包覆纳米铁 或铁碳化合物纳米颗粒 ${ }^{[9]}$, 呈球形或椭球形，分 散性较好, 但颗粒大小不均匀。从图 5 中 C-1 与 C-2 发现, 碳包覆铁纳米颗粒尺寸较图 5B-2 样品 的小, 且大小相对均匀, 但分散性有所下降。因此, 随着二茂铁质量的增加，合成的颗粒趋向均匀化， 二茂铁质量为 $3.5 \mathrm{~g}$ 较 $2.5 \mathrm{~g}$ 制备的颗粒均匀, 且 包覆也较好。

通过高分辨率透射电镜对爆轰产物的微观形貌 进一步分析, 从图 6 可看出, $\mathrm{A}$ 图样品的核壳结构不 明显, $\mathrm{B} 、 \mathrm{C}$ 图样品均可明显地看出碳层, 颜色较深 处为金属核。 $\mathrm{B}$ 图中碳层厚度约为 $5 \mathrm{~nm}, \mathrm{C}$ 图样品 中碳层厚度约 $10 \mathrm{~nm}$, 且二者石墨化程度较高。
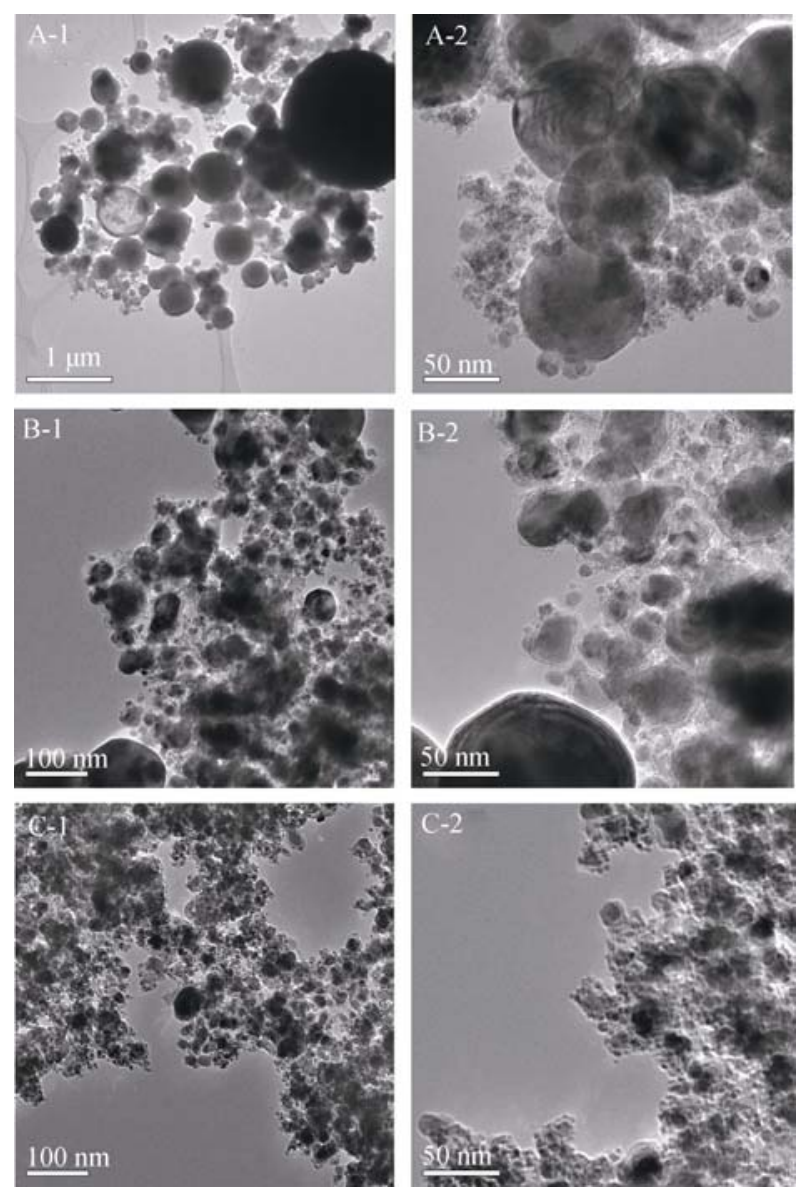

图 5 不同二茂铁质量获得产物的 TEM 照片

Fig. 5 TEM images of detonation products with different ferrocene masses

Ferrocene mass: A) $1.5 \mathrm{~g}$; B) $2.5 \mathrm{~g}$; C) $3.5 \mathrm{~g}$ 

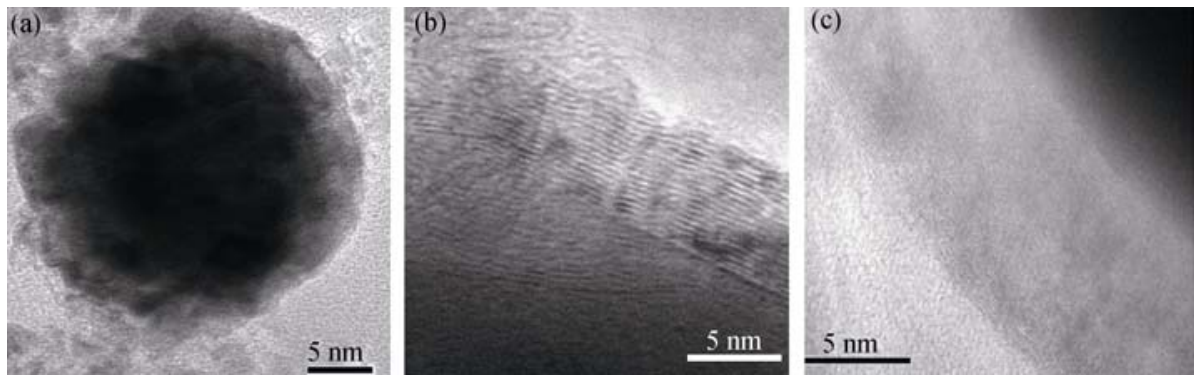

图 6 不同二茂铁质量制备产物的 HR-TEM 照片

Fig. 6 HR-TEM images of detonation products prepared with different ferrocene masses Ferrocene mass: (a) $1.5 \mathrm{~g}$; (b) $2.5 \mathrm{~g}$; (c) $3.5 \mathrm{~g}$

\section{3 结论}

1) 氢氧比例与二茂铁质量在合成碳包铁纳米 颗粒时相互影响。当氢氧比例为 $2: 1$, 二茂铁质量在 2.5 3.5 g 时, 能够合成碳包覆铁纳米颗粒。并且二 茂铁为 $3.5 \mathrm{~g}$, 氢氧比例为 $2: 1$ 时, 制备出的碳包铁 纳米颗粒具有大小较均匀、形貌结构良好及分散性 较高特点。因此，该方案是制备碳包铁的较佳方案。

2) 二茂铁质量对能否合成碳包铁纳米颗粒影 响很大：当其质量过少时，导致铁元素部分被氧化; 当其质量过多时，则因其无法被完全分解，而产生 杂质。

由于本研究仍处于探索阶段, 其中影响实验结 果的其他因素(爆压、爆速等)将在以后的工作中作 进一步的研究。

\section{参考文献:}

[1] BYSTRZEJEWSKI M, PYRZYNSKA K, HUCZKO A, et al. Carbon-encapsulated magnetic nanoparticles as separable and mobile sorbents of heavy metal ions from aqueous solutions. Carbon, 2009, 47(4): 1201-1204.

[2] WANG Y, WEI W, MASPOCH D, et al. Superparamagnetic sub-5 nm $\mathrm{Fe} @ \mathrm{C}$ nanoparticles: isolation, structure, magnetic properties, and directed assembly. Nano Letters, 2008, 8(11): 3761-3765.

[3] PARK J B, JEONG S H, JEONG M S, et al. Synthesis of carbonencapsulated magnetic nanoparticles by pulsed laser irradiation of solution. Carbon, 2008, 46(11): 1369-1377.

[4] GRUDZINSKI I P, BYSTRZEJEWSKI M, CYWINSKA M A, et al. Assessing carbon-encapsulated iron nanoparticles cytotoxicity in Lewis lung carcinoma cells. Journal of Applied Toxicology, 2014, 34(4): 380-394.

[5] BORYSIUK J, GRABIAS A, SZCZYTKO J, et al. Structure and magnetic properties of carbon encapsulated $\mathrm{Fe}$ nanoparticles ob tained by arc plasma and combustion synthesis. Carbon, 2008, 46(13): 1693-1701.

[6] XU B, GUO J, WANG X, et al. Synthesis of carbon nanocapsules containing $\mathrm{Fe}$, Ni or $\mathrm{Co}$ by arc discharge in aqueous solution. Carbon, 2006, 44(13): 2631-2634.

[7] SERGIIENKO R, SHIBATA E, AKASE Z, et al. Carbon encapsulated iron carbide nanoparticles synthesized in ethanol by an electric plasma discharge in an ultrasonic cavitation field. Materials Chemistry and Physics, 2006, 98(1): 34-38.

[8] WANG S, HUANG X, HE Y, et al. Synthesis, growth mechanism and thermal stability of copper nanoparticles encapsulated by multi-layer graphene. Carbon, 2012, 50(6): 2119-2125.

[9] WU W Z, ZHU Z P, LIU Z Y, et al. Preparation of carbonencapsulated iron carbide nanoparticles by an explosion method. Carbon, 2003, 41(2): 317-321.

[10] NING L, XIAOJIE L, XIAOHONG W, et al. Preparation and magnetic behavior of carbon-encapsulated iron nanoparticles by detonation method. Composites Science and Technology, 2009, 69(15/16): 2554-2558.

[11] LI X, LUO N, YAN H, et al. Preparation of carbon-encapsulated ferronickel nanoparticles by detonation method and its characterization. Rare Metal Materials and Engineering, 2010, 391: 429-433.

[12] LUO N, LI X, WANG X, et al. Detonation synthesis of carbonencapsulated nickel/cobalt nanoparticles. Explosion and Shock Waves, 2010, 30: 390-394.

[13] LUO N, LI X, YAN H, et al. Detonation synthesis of carbon encapsulated cobalt/nickel nanoparticles. Chinese Journal of High Pressure Physics, 2009, 23(6): 415-420.

[14] LI X, ZHANG X, LUO N, et al. Synthesis of carbon-encapsulated copper nanoparticles by detonation method. Explosion and Shock Waves, 2012, 32(2): 174-178. 